\title{
Unidades de soporte vital básico y avanzado en España: análisis de la situación actual
}

\author{
Basic and advanced life support units in Spain: \\ analysis of the current situation
}

\section{S. Ballesteros}

\section{RESUMEN}

Fundamento. Describir el tipo y número de ambulancias asistenciales de urgencia integradas en el Sistema de Emergencias Médicas de cada comunidad autónoma en España y calcular su relación con la población a la que asisten.

Metodología. Se ha revisando la bibliografía oficial de cada Comunidad Autónoma y se han extraído los datos referentes al tipo y número de recursos asistenciales terrestres con capacidad de realizar traslado de pacientes integradas en el Sistema de Emergencias Médicas y datos poblacionales, correspondientes a 2011. Se excluyeron los recursos destinados al transporte secundario o especializado y aquellos activos a tiempo parcial computaron de manera proporcional al tiempo de operatividad.

Resultados. Se han identificado un total de 319 Unidades de Soporte Vital Avanzado (USVA) y 1.109,05 Unidades de Soporte Vital Básico (USVB), de las que 31,9 están provistas con personal de enfermería. La tasa estatal de ambulancias por población absoluta ha resultado de 0,86 USVA (rango 0,32-1,44) y 2,35 USVB (rango $0,07-5,30$ ) por cada 100.000 habitantes. La tasa estatal por población relativa se ha estimado en 0,29 (rango 1,06-30,22) y 0,08 (rango $0,94-16,03$ ) habitantes $/ \mathrm{km}^{2}$ por USVA y USVB respectivamente y la tasa nacional por superficie en 1586,2 (rango $140,8-4204,5$ ) y 456,2 (rango $44,1-14599,6) \mathrm{km}^{2}$ por USVA y USVB respectivamente.

Conclusiones. Existen grandes diferencias en cuanto a los índices calculados de los recursos asistenciales de emergencias de las comunidades autónomas sobre su población y extensión, no estableciéndose, además, relación con la idiosincrasia geodemográfica de cada territorio. Estas diferencias podrían ser causadas por una heterogeneidad en la gestión de los SEM u otros factores no contemplados en este trabajo.

Palabras clave. Urgencias médicas. Sistema Médico de Emergencia. Medios movilizables en casos de emergencia. España.

\begin{abstract}
Background. To describe the type and number of emergency care ambulances integrated in the Emergency Medical System in the Spanish autonomous communities and to calculate their relationship with the assigned population.

Material and methods. The official literature of the autonomous communities was reviewed, and the type and number of terrestrial care resources integrated in the Emergency Medical System and population data for 2011 were extracted. Secondary or specialized resources were excluded, and part-time units were computed in proportion to operational time.
\end{abstract}

Results. A total of 319 Advanced Life Support Units (ALSU) and 1,109.05 Basic Life Support Units (BLSU) were identified, of which 31.9 are provided with nursing staff. The national rate of ambulances by absolute population was 0.86 ALSU (range 0.32 to 1.44 ) and 2.35 BLSU (range 0.07 to 5.30 ) per 100,000 inhabitants. The state rate by relative population was estimated at 0.29 (range 1.06 to 30.22 ) and 0.08 (range 0.94 to 16.03 ) inhabitants $/ \mathrm{km}^{2}$ for ALSU and BLSU respectively, and the national rate by surface was $1,586.2$ (range 140.8 to 4204.5 ) and $456.2 \mathrm{~km}^{2}$ (range 44.1 to 14599.6) for ALSU and BLSU respectively.

Conclusions. There are significant differences in the calculated rates of emergency care resources of autonomous regions over their population and area. No relation was found with the geodemographic idiosyncrasies of each territory. These differences could be caused by heterogeneity in the management of EMS or other factors not considered in this work.

Key words. Emergencies. Emergency Medical System. Mobile Media in Emergency Cases. Spain.

\section{Correspondencia:}

S. Ballesteros Peña

Parque de Bomberos de Garellano

C/ Luis Briñas 16

48013 Bilbao (Bizkaia)

Email: sendoa.ballesteros@gmail.com
Aceptación provisional: 24 de febrero de 2012

Aceptación definitiva: 5 de marzo de 2012 


\section{INTRODUCCIÓN}

En 1984 la Sociedad Española de Medicina Intensiva elabora el Plan de Actuación Sanitaria de Urgencia, sentando las bases para la creación de un sistema de asistencia médica de urgencia ${ }^{1}$. Sin embargo, no es hasta la década de los 90 cuando se terminan de implantar los equipos de emergencias extrahospitalarios, desarrollados a partir de las recomendaciones del Comité Europeo de Salud presentadas al Consejo de Europa en $1988^{2}$.

Desde el año $2006^{3}$, el modelo organizativo de la atención urgente en España queda asumido por las administraciones sanitarias de cada comunidad autónoma, siendo premisas comunes de su cartera de servicios la realización de la prestación en el tiempo y lugar adecuado para facilitar un tratamiento adaptado a las necesidades de los pacientes, garantizando una asistencia integral dentro del Sistema de Emergencias Médicas (SEM). Además, se establecieron los centros de coordinación de urgencias como estructuras básicas para gestionar la accesibilidad y coordinación de los recursos intervinientes durante las 24 horas del día.

De esta manera, conviven en España diferentes modelos organizativos de los SEM que, aún teniendo muchas similitudes, presentan importantes diferencias en cuanto a las características de los centros de coordinación, denominación de profesionales o vehículos, equipamientos, grado de desarrollo y distribución.

El objetivo de este estudio se centra en determinar el número y tipología de ambulancias asistenciales de emergencias primaras integradas en el SEM de cada comunidad autónoma en España y calcular sus ratios en función de la población y extensión que cubren.

\section{METODOLOGÍA}

De la revisión de la bibliografía oficial de cada comunidad autónoma disponible en webs oficiales, boletines informativos o memorias anuales ${ }^{4-17}$ se extrajeron datos referidos al tipo y número de vehículos asistenciales de urgencia disponibles a fe- cha de junio de 2011. Cuando fue preciso se contactó directamente (vía telefónica o email) con las entidades proveedoras del servicio o asociaciones profesionales en busca de información actualizada.

Los datos relativos a la superficie de cada comunidad fueron extraídos del Instituto Nacional de Estadística ${ }^{18}$ y los poblacionales fueron obtenidos de la última revisión del Padrón municipal, a 1 de enero de 2011, realizada por el mismo organismo ${ }^{19}$.

Se incluyeron en el estudio todos los vehículos terrestres de Soporte Vital Avanzado (USVA) y Soporte Vital Básico (USVB), o con personal de enfermería, con capacidad de trasladar y asistir en ruta a un paciente, integrados en los Sistemas de Emergencias Médicas de cada comunidad autónoma.

De acuerdo con la legislación vigente en materia de transporte sanitario por carretera $^{20}$, se han clasificado como USVA a aquellos recursos asistenciales dotados del equipamiento técnico propio para realizar tareas de soporte vital avanzado y un personal mínimo de la categoría médicos, enfermeros y técnicos en emergencias sanitarias, y como USVB a los orientados a la prestación de un apoyo vital básico/instrumentalizado y un equipo mínimo conformado por dos técnicos en emergencias o técnico y enfermero.

Fueron excluidos los recursos destinados al transporte secundario (interhospitalario), los servicios especializados (transporte pediátrico o psiquiátrico) y las unidades no asistenciales. Aquellos activos a tiempo parcial o como refuerzo estacional computaron de manera proporcional al tiempo de operatividad (por ejemplo, un recurso operativo únicamente durante 12 horas al día se computaría como 0,5 ).

La proporción de unidades con respecto a la población absoluta se expresa en forma de ratio, como número de recursos por 100.000 habitantes. Los ratios de ambulancias en función de la población relativa (densidad de población) se presentan en forma de habitantes $/ \mathrm{km}^{2}$ por recurso y como kilómetros cuadrados por unidad para representar la distribución por extensión territorial. 
Además se calcularon los valores medios de los índices de todas las comunidades autónomas, con sus respectivos intervalos de confianza del 95\% (IC 95\%). En esta operación se excluyeron, por sus condiciones geodemográficas especiales, las ciudades autónomas de Ceuta y Melilla.

Para el proceso y análisis de datos se emplearon los programas PASW 17 y Excel 2007.

\section{RESULTADOS}

Siguiendo el sistema de cómputo propuesto, se han contabilizado 319 recursos de Soporte Vital Avanzado y 1.109,05 de Soporte Vital Básico, lo que representa un ratio de una USVA por cada 3,5 USVB. Es de reseñar que 31,9 de las unidades de SVB integran personal de enfermería (USVE): 5 en Andalucía, 4 en Canarias, 16 en Cataluña (de las que 5 funcionan a 12 horas) y 10 en País Vasco (con 2 unidades no activas los fines de semana).

Su distribución por comunidades autónomas y provincias, así como sus características geodemográficas, se detalla en la tabla 1.

Expresado en índices demográficos, la tasa estatal de unidades de emergencia por 100.000 habitantes ha resultado de 0,68 para las USVA y de 2,35 para las USVB (valores promedio de todas las comunidades autónomas de 0,77; IC 95\% 0,62-0,92 y 2,56; IC $95 \%$ 1,85-3,27, para USVA y USVB respectivamente). Sin embargo se objetivan grandes diferencias regionales, con ratios que oscilan entre 0,32-0,33 (Andalucía y Galicia) de mínima y 1,44 (Extremadura) de máxima para las USVA y entre 0,07 (Andalucía) y 5,30 (Navarra) en las USVB (Fig. 1). Extremadura no cuenta en su dispositivo con USVB.

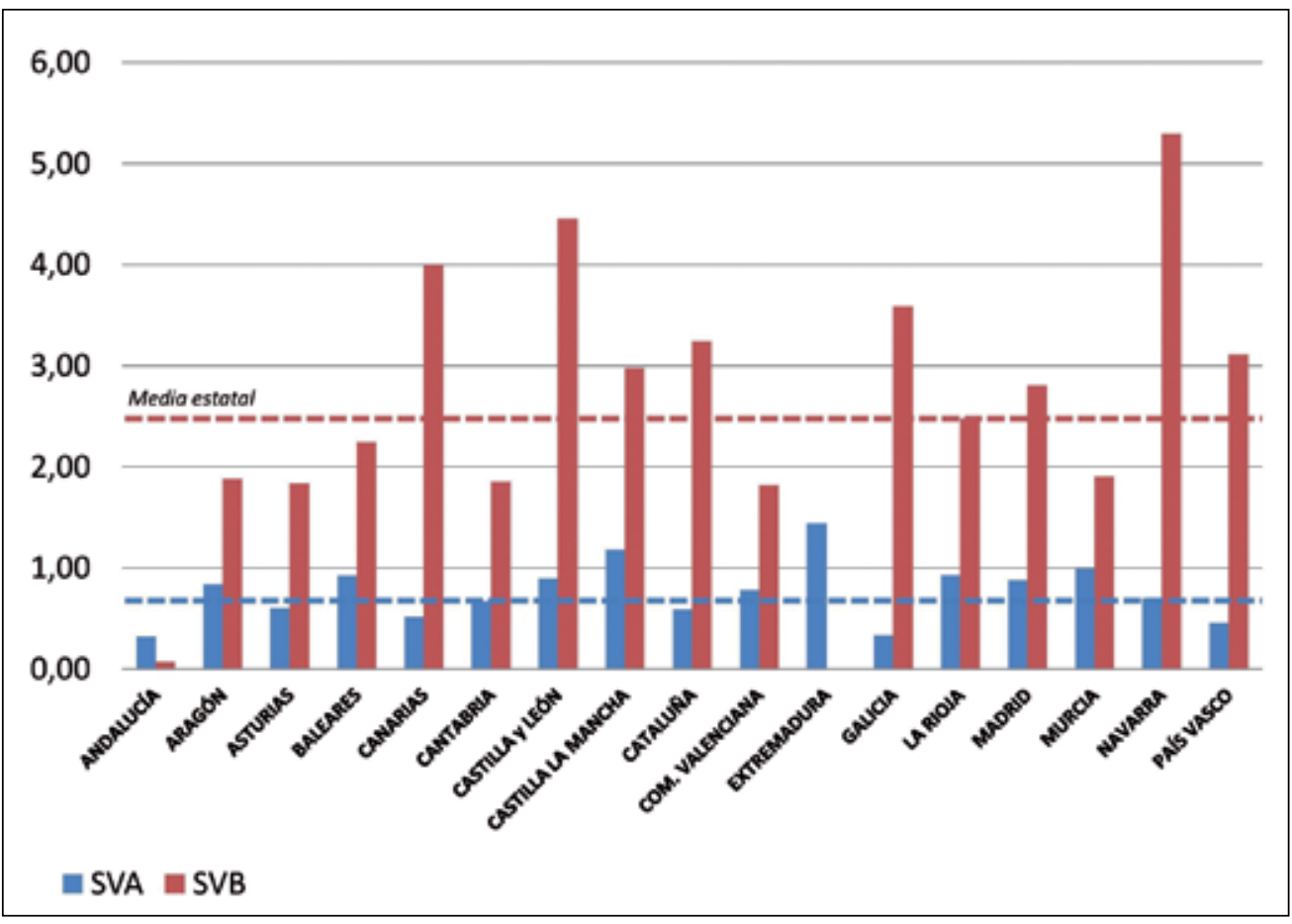

Figura 1. Índices de recursos de Soporte Vital Básico y Avanzado según la población absoluta en las diferentes comunidades autónomas (unidades asistenciales por 100.000 habitantes). 
Tabla 1. Características geodemográficas de las provincias de España y distribución de las unidades de emergencia asumidas por los Sistemas de Emergencias Médicas

\begin{tabular}{|c|c|c|c|c|c|c|}
\hline Comunidad & Provincia & Habitantes & $\begin{array}{c}\text { Superficie } \\
\left(\mathrm{Km}^{2}\right)\end{array}$ & USVA & USVB & USVE \\
\hline \multirow[t]{9}{*}{ ANDALUCÍA } & & 8424102 & 87597,69 & 27 & 1 & 5 \\
\hline & Almería & 702819 & 8774,87 & 2 & & \\
\hline & Cádiz & 1243519 & 7435,88 & 2 & & \\
\hline & Córdoba & 805857 & 13771,31 & 3 & & \\
\hline & Granada & 924550 & 12646,98 & 3 & & \\
\hline & Huelva & 521968 & 10128,01 & 2 & & \\
\hline & Jaén & 670600 & 13496,09 & 2 & & \\
\hline & Málaga & 1625827 & 7308,46 & 7 & & 3 \\
\hline & Sevilla & 1928962 & 14036,09 & 6 & 1 & 2 \\
\hline \multirow[t]{4}{*}{ ARAGÓN } & & 1346293 & 47720,59 & 11,35 & 25 & \\
\hline & Huesca & 228361 & 15636,4 & 4 & 7 & \\
\hline & Teruel & 144607 & 14809,57 & 3 & 6 & \\
\hline & Zaragoza & 973325 & 17274,62 & 4,35 & 12 & \\
\hline ASTURIAS & & 1081487 & 10602,41 & 6,5 & 19,85 & \\
\hline BALEARES & & 1113114 & 4991,66 & 10,3 & 25 & \\
\hline \multirow[t]{3}{*}{ CANARIAS } & & 2126769 & 7446,95 & 11 & 81 & 4 \\
\hline & Las Palmas & 1096980 & 4065,78 & 6 & 41 & 2 \\
\hline & Tenerife & 1029789 & 3381,17 & 5 & 40 & 2 \\
\hline CANTABRIA & & 593121 & 5321,34 & 4 & 11 & \\
\hline \multirow[t]{10}{*}{ CASTILLA Y LEÓN } & & 2558463 & 94226,53 & 23 & 114 & \\
\hline & Ávila & 172704 & 8049,92 & 2 & 12 & \\
\hline & Burgos & 375657 & 14291 & 4 & 17 & \\
\hline & León & 497799 & 15581,66 & 3 & 22 & \\
\hline & Palencia & 171668 & 8052,49 & 2 & 8 & \\
\hline & Salamanca & 352986 & 12349,95 & 3 & 15 & \\
\hline & Segovia & 164169 & 6922,75 & 1 & 10 & \\
\hline & Soria & 95223 & 10307,17 & 1 & 8 & \\
\hline & Valladolid & 534874 & 8110,33 & 4 & 11 & \\
\hline & Zamora & 193383 & 10561,26 & 3 & 11 & \\
\hline \multirow[t]{6}{*}{ CASTILLA MANCHA } & & 2115334 & 79461,97 & 25 & 63 & \\
\hline & Albacete & 402318 & 14925,84 & 5 & 13 & \\
\hline & Cuenca & 219138 & 19813,23 & 3 & 14 & \\
\hline & Ciudad Real & 530175 & 17141,13 & 7 & 14 & \\
\hline & Guadalajara & 256461 & 12212,09 & 3 & 11 & \\
\hline & Toledo & 707242 & 15369,68 & 7 & 11 & \\
\hline
\end{tabular}


UNIDADES DE SOPORTE VITAL BÁSICO Y AVANZADO EN ESPAÑA: ANÁLISIS DE LA SITUACIÓN ACTUAL

\begin{tabular}{|c|c|c|c|c|c|c|}
\hline Comunidad & Provincia & Habitantes & $\begin{array}{c}\text { Superficie } \\
\left(\mathbf{K m}^{2}\right)\end{array}$ & USVA & USVB & USVE \\
\hline \multirow[t]{5}{*}{ CATALUÑA } & & 7539618 & 32113,4 & 44,5 & 230,45 & 13,5 \\
\hline & Barcelona & 5529099 & 7728,17 & 23 & 127,8 & 11 \\
\hline & Girona & 756810 & 5909,88 & 6 & 34,4 & 1 \\
\hline & Lléida & 442308 & 12172,49 & 9,5 & 24,5 & 0,5 \\
\hline & Tarragona & 811401 & 6302,86 & 6 & 43,75 & 1 \\
\hline CEUTA & & 82376 & 19,48 & 1 & 1 & \\
\hline \multirow[t]{4}{*}{ COM. VALENCIANA } & & 5117190 & 23254,52 & 40 & 93 & \\
\hline & Alicante & 1934127 & 5816,58 & 15,5 & 36,5 & \\
\hline & Castellón & 604344 & 6631,85 & 8 & 16 & \\
\hline & Valencia & 2578719 & 10806,09 & 16,5 & 40,5 & \\
\hline \multirow[t]{3}{*}{ EXTREMADURA } & & 1109367 & 41634,48 & 16 & & \\
\hline & Badajoz & 693921 & 21766,28 & 6 & & \\
\hline & Cáceres & 415446 & 19868,2 & 7 & & \\
\hline \multirow[t]{5}{*}{ GALICIA } & & 2795422 & 29574,86 & 9,25 & 100,25 & \\
\hline & A Coruña & 1147124 & 7950,42 & 4 & 36 & \\
\hline & Lugo & 351530 & 9856,56 & 1 & 20 & \\
\hline & Ourense & 333257 & 7273,22 & 1 & 15 & \\
\hline & Pontevedra & 963511 & 4494,66 & 3,25 & 29,25 & \\
\hline LA RIOJA & & 322955 & 5045,32 & 3 & 8 & \\
\hline MADRID & & 6489680 & 8027,69 & 57 & 182 & \\
\hline MELILLA & & 78476 & 13,41 & 1 & 2 & \\
\hline MURCIA & & 1470069 & 11313,16 & 14,6 & 28 & \\
\hline NAVARRA & & 642051 & 10390,41 & 4,5 & 34 & \\
\hline \multirow[t]{4}{*}{ PAÍS VASCO } & & 2184606 & 7230,03 & 10 & 58,6 & 9,4 \\
\hline & Araba & 319227 & 3032,4 & 1 & 11 & 1 \\
\hline & Bizkaia & 1155772 & 2217,28 & 5 & 25,6 & 7,4 \\
\hline & Gipuzkoa & 709607 & 1980,35 & 4 & 22 & 1 \\
\hline TOTAL ESPAÑA & & 47190493 & 505985,9 & 319 & 1077,15 & 31,9 \\
\hline
\end{tabular}

USVA: Unidades de Soporte Vital Avanzado. USVB: Unidades de Soporte Vital Básico. USVE: Unidades de Soporte Vital con Enfermería. 
La figura 2 muestra la proporción de unidades según la población relativa de cada comunidad autónoma o el número de habitantes por kilómetro cuadrado que atiende cada unidad asistencial. Las tasas estatales se establecen en 0,29 y 0,08 habitantes/kilómetro cuadrado por cada USVA y USVB respectivamente (valores medios de 12,38 ; IC $95 \%$ 7,27-17,5 y 4,56; IC $95 \%$ 2,25-6,87).

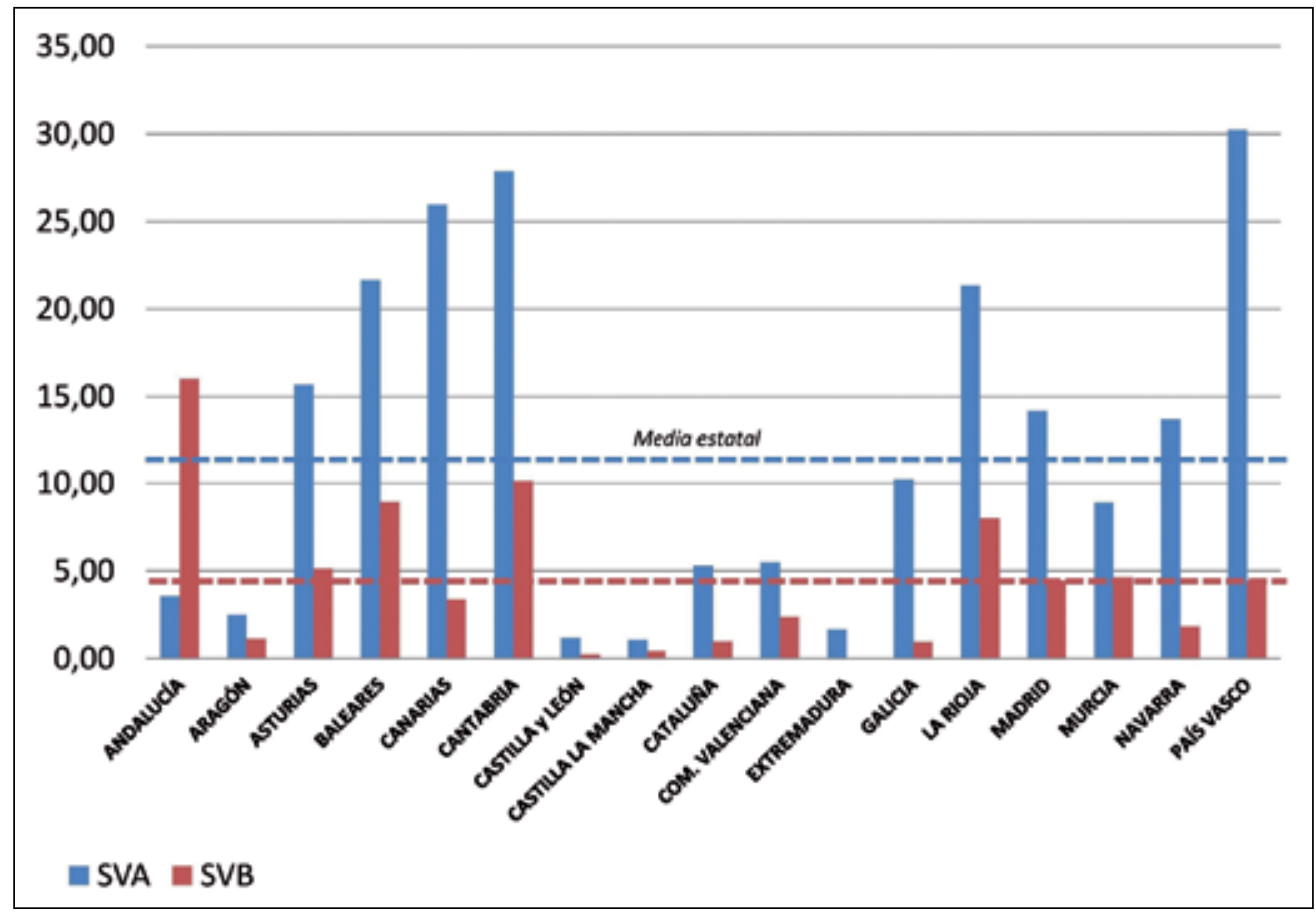

Figura 2. Índices de recursos de Soporte Vital Básico y Avanzado según la población relativa de cada comunidad autónoma (habitantes por kilómetro cuadrado y unidad asistencial).

Desde el punto de vista de los índices geográficos, la tasa estatal se establece en $1586,2 \mathrm{~km}^{2} /$ USVA y $456,2 \mathrm{~km}^{2} /$ USVB (valores medios de todas las comunidades autónomas de 1.857,6; IC 95\% 1.168,89-2.546,31 y 1.298,16; IC 95\% -482,07-3.078,39, para USVA y USVB), pudiendo observarse importantes variaciones entre territorios, y destacando la comunidad de Madrid, al presentar la relación más baja entre superficie y recurso, tanto en el caso de las USVA como de las USVB (Fig. 3).

Las ciudades autónomas de Ceuta y Melilla, dadas sus peculiaridades geodemográficas presentan índices muy alejados de la tónica de los territorios insulares. 


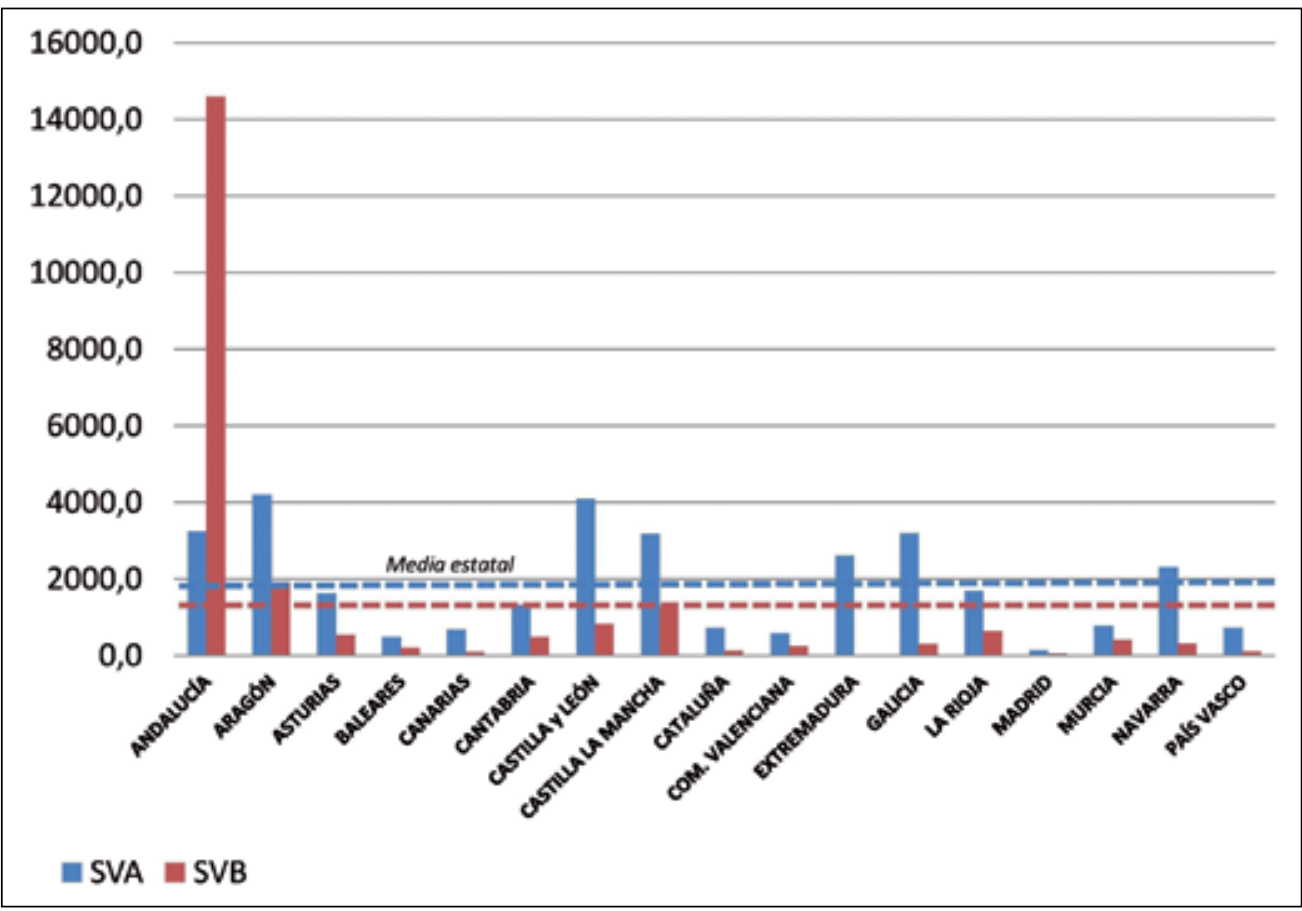

Figura 3. Índices de recursos de Soporte Vital Básico y Avanzado por superficie de las comunidades autónomas (kilómetros cuadrados por unidad asistencial).

\section{DISCUSIÓN}

España es uno de los países de la Unión Europea con mayor distancia entre el domicilio y el hospital de referencia ${ }^{21}$, lo que convierte al transporte sanitario en una actividad crucial dentro de la prestación sanitaria.

Es por ello que los SEM constituyen un elemento importante en los servicios de salud en todos los países de la Unión Europea. Además, en las últimas décadas han tomado especial protagonismo, al haberse producido un incremento generoso en la demanda de atención urgente, posiblemente relacionado con el aumento de las expectativas y de la edad de la población.

Todos los ciudadanos españoles tienen derecho a recibir el mismo nivel y calidad de atención sanitaria urgente ${ }^{22}$, por lo que es necesario que las administraciones públicas realicen un esfuerzo para dotar a las comunidades autónomas de los recursos necesarios de acuerdo a una serie de criterios objetivos. El número de unidades de emergencias por habitante es uno de los indicadores recomendados en la evaluación de la calidad de la atención sanitaria extrahospitalaria ${ }^{23}$, presentándose útil para la monitorización ${ }^{24}$ de los sistemas de emergencia y comparación entre administraciones.

Los índices de ambulancia por población absoluta (número de recursos asistenciales por 100.000 habitantes) vienen a representar el nivel de cobertura ofrecido por sistema de emergencias. Un índice alto proporciona una mayor garantía para que en el caso de surgir dos o varias emergencias al mismo tiempo pueda asignarse el recurso más adecuado.

Por otro lado, los índices según la población relativa (habitantes por kilómetro cuadrado y unidad asistencial) pueden ser 
representativos de la dispersión de la población que atiende una ambulancia.

Finalmente, los índices representativos de la superficie de cobertura de una unidad (kilómetros cuadrados por unidad asistencial) reflejan la distancia teórica que debería recorrer una ambulancia para ofrecer atención sanitaria, lo que se traduce en un mayor tiempo de respuesta cuanto más elevado resulta el índice calculado.

$\mathrm{Al}$ contrastar los datos calculados entre territorios se objetiva una evidente disparidad de resultados. Sin embargo, la comparación entre comunidades en función de los diferentes índices obtenidos es un proceso que debe ser realizado con cierta cautela, ya que no se ha evidenciado una clara relación entre los resultados calculados y las cifras poblaciones o extensión de cada territorio. Este hallazgo debe orientarnos a pensar que las variaciones puedan deberse a criterios de gestión y organización sanitaria autonómica.

Por esa razón, es posible localizar provincias que no contemplan en su sistema de emergencias a los recursos de SVB (caso de Extremadura) o lo hacen de manera discreta (Andalucía). O la profusión de respuestas intermedias, utilizando unidades menos complejas, potenciando el papel autónomo del personal de enfermería en las USVE (como en los casos de Andalucía, Canarias, Cataluña y País Vasco).

Mención merece también la utilización programada en algunas comunidades de ambulancias de refuerzo en periodos de máxima confluencia de población (por turismo estival, como en Galicia o Levante) o ante la previsión de aumento de patologías de carácter cíclico o epidémico (como el Plan Integral de Urgencias de Cataluña).

Algunos autores ${ }^{25}$ han recomendado la posibilidad de utilizar estos indicadores de una forma práctica, afirmando que encontrarse por debajo del valor medio estatal calculado en una sola de las proporciones consideradas (población o extensión) puede ser consecuencia de la idiosincrasia del territorio, pero, el hecho de encontrarse alejado del valor promedio en todos los indicadores debe ser motivo de reflexión y estudio pormenorizado.
No obstante, recientes recomendaciones ${ }^{26}$ se centran en el empleo de otros índices más pragmáticos, aunque también de más complejo cálculo, fundamentándose en criterios de isócrona, estableciendo además estándares de calidad. Esta sistemática presenta la capacidad de considerar indirectamente algunas peculiaridades territoriales (orografía, vías de comunicación...).

Los datos numéricos de este trabajo muestran datos actualizados a fecha de junio de 2011. No obstante, es en julio del mismo año cuando la Consejería de Salud de Cataluña inicia una serie de recortes económicos encuadrados en un plan de austeridad y simplificación de la Administración Sanitaria ${ }^{27}$ que han afectado directamente al sector del transporte sanitario, mermando el número de horas de operatividad de la flota de ambulancias que compone su red de emergencias. Esta medida, y la posibilidad de otras que en distintas comunidades se pronuncien en el mismo sentido, propiciarán ligeras variaciones con respecto al cómputo de unidades de emergencia estimable de cara a 2012.

A la vista de los resultados obtenidos cabe concluir que, entre las comunidades autónomas de España, se perciben grandes diferencias en cuanto a los índices calculados de sus recursos asistenciales de emergencias sobre su población y extensión. Además, estas variaciones no se relacionan con la idiosincrasia geodemográfica de cada territorio, por lo que deben ser atribuidas a una heterogeneidad de criterios en la gestión de los SEM u otros factores no contemplados en este trabajo.

\section{BIBLIOGRAFÍA}

1. Perales N. Avances en emergencias y resucitación. Edikamed. 1996

2. Barroeta J, BoAda N. Los servicios de emergencia y urgencias médicas extrahospitalarias en España. MENSOR. 2011

3. ReAl Decreto 1030/2006, DE 15 DE SEPTIEMBRE DE 2006 , por el que se establece la cartera de servicios comunes del sistema nacional de Salud y el procedimiento para su actualización. Boletín Oficial del Estado 222, del 16 de 
septiembre de 2006. (Consultado el 1 diciembre 2011). Disponible en: http://www.boe.es/ boe/dias/2006/09/16/pdfs/A32650-32679.pdf

4. Instituto de Información SanitaRia. Atención a la urgencia extrahospitalaria. Organización en las comunidades autónomas. Sistema de Información de Atención Primaria (SIAP). 2010 [Publicación en Internet]. Madrid: Ministerio de Sanidad, Política Social e Igualdad; 2011. (Consultado 23 de diciembre de 2011). Disponible en: http://www.mspsi.es/ estadEstudios/estadisticas/estadisticas/estMinisterio/siap.htm

5. Gobierno Vasco. Departamento de Sanidad. Osakidetza-Servicio Vasco de Salud. Emergencias: Nuestros Recursos. [WEBSITE] (Consultado $15 \mathrm{de}$ diciembre de 2011). Disponible en: http://www. osakidetza.euskadi.net/r85-gkemer02/es/contenidos/informacion/emer_nuestros_recursos/es_emer/emergencias_nuestros_recursos. html

6. Gobierno de Cantabria. Consejería de Sanidad. Servicio Cántabro de Salud. 061: recursos 061. [WEBSITE] (Consultado 23 de diciembre de 2011). Disponible en: http://www.scsalud. es/061/recursos061/index.php

7. Xunta de Galicia. Consellería de Sanidade. Sevizio Galego de Saúde. Fundación Pública Urgencias Sanitarias de Galicia-061. Recursos técnicos. [WEBSITE] (Consultado 3 de enero de 2012). Disponible en: http://061.sergas.es/asp/inicio.asp?idioma $=1$

8. Gobierno de Aragón. Departamento de Salud y Consumo. Servicio Aragonés de Salud. 061 Aragón - Urgencias y emergencias sanitarias. [WEBSITE] (Consultado 3 de enero de 2012). Disponible en: http://www.aragon.es/DepartamentosOrganismosPublicos/Organismos/ ServicioAragonesSalud/AreasTematicas/ InformacionCiudadano_DerechosDeberes/ ci.09_aragon_urgencias.detalleDepartament $\mathrm{o}$ ?channelSelected $=5 \mathrm{~b} 39 \mathrm{dd} 2 \mathrm{a} 38 \mathrm{e} 2 \mathrm{~b} 210 \mathrm{VgnV}$ CM100000450a15acRCRD

9. Ayuntamiento de Zaragoza. Consejería de Servicios Públicos. Bomberos y Protección Civil. AsisTENCIA Médica. [WEBSITE] (Consultado 25 de diciembre de 2011). Disponible en: http:// www.zaragoza.es/ciudad/bomberos/asistencia/componentes.htm

10. Gobierno del Principado de Asturias. Servicio de Salud del Principado de Asturias. Astursalud. Recursos y Organigrama del SAMU. [WEBSITE] (Consultado 23 de diciembre de 2011). Disponible en: http://www.asturias.es/por$\mathrm{tal} /$ site/astursalud/menuitem.2d7ff2df00b62 $567 \mathrm{dbdfb} 51020688 \mathrm{a} 0 \mathrm{c} /$ ?vgnextoid=b748d781 ddb43210VgnVCM10000097030a0aRCRD
11. Govern de les Illes Balears. Servei d’Emergències DE les Illes Balears - SEIB 112. [WEBSITE]. (Consultado 23 de diciembre de 2011). Disponible en: http://www.caib.es/sacmicrofront/contenido.do?mkey=M08082109010018 $816268 \&$ lang $=$ ca\&cont $=8673$

12. Consejería de SANidAd y Consumo de la Región de Murcia. Gerencia de EMERGencias 061 [WEBSITE]. (Consultado el 23 de diciembre de 2011. Disponible en: http://www.061murcia.org/

13. Comunidad de Madrid. Servicio de urgencia Médica DE MADRID - SUMMA112. Medios. [WEBSITE]. (Consultado 23 de diciembre de 2011). Disponible en: http://www.madrid.org/cs/Satell ite?language $=$ es \&pagename $=$ SUMMA $112 \% 2 \mathrm{~F}$ Page\%2FS112_home

14. Ayuntamiento de Madrid. Emergencias y Seguridad. SAMUR PRotección CIVL. [WEBSITE]. (Consultado 23 de diciembre de 2011). Disponible en: http://www.madrid.es/portal/site/munimadrid/menuitem.f4bb5b953cd0b0aa7d245f019f c08a0c/?vgnextoid=c88fcdb1bfffa010VgnVCM 100000d90ca8c0RCRD\&vgnextchannel=d11c9 ad016e07010VgnVCM100000dc0ca8c0RCRD\& vgnextfmt $=$ default $\&$ rmColeccion $=6 \mathrm{eb} 210200 \mathrm{a}$ b59110VgnVCM1000000b205a0aRCRD

15. Junta de Andalucía. Conserjería de Salud. Empresa Pública de Emergencias Sanitarias - EPES 061. [WEBSITE]. (Consultado el 24 de diciembre de 2011). Disponible en: http://www.epes.es/ cocoon/epes-estaticoImprimir-.html?langua ge $=\& \mathrm{c}=$ recursosAsistenciales

16. Junta de Castilla y León. Conserjería de Sanidad SACYL. EmERGENCIAS SANitARIAS EN CASTILLA Y LeÓN. [WEBSITE]. (Consultado el 24 de diciembre de 2011). Disponible en: http://www.saludcastillayleon.es/ciudadanos/es/urgenciasemergencias/emergencias-sanitarias-castilla-leon

17. Generalitat de Catalunya. Departament de SaLUT. Recursos SEM. Distribución territorial. [WEBSITE]. (Consultado 29 de diciembre de 2011). Disponible en: http://www20.gencat.cat/docs/salut/Minisite/SEM/SEM $\% 20$ Emerg\%C3\%A8ncies\%20M\%C3\%A8diques/ Documents/Arxius/Distribucio\%20territorial\%20CAT.pdf

18. Instituto Nacional de Estadística. Territorio. Población, superficie y densidad por CCAA y provincias. [WEBSITE] (Consultado 23 diciembre de 2011). Disponible en: http:// www.ine.es/jaxi/tabla.do?path=/t43/a011/ a1998/densidad/a2010/10/\&file=t 10031 . px\&type $=$ pcaxis $\& L=0$

19. Instituto Nacional de Estadística. Cifras de Población referidas al 01/01/2011. REAL 
DECRETO 1782/2011, de 26 de diciembre: poblaciones referidas al 1 de enero de 2008 por comunidades autónomas y sexo. [WEBSITE] (Consultado 23 diciembre de 2011). Disponible en: http://www.ine.es/jaxi/tabla. do? path=/t20/e260/a2011/10/\&file=pro001. px\&type $=$ pcaxis \&L=

20. Real Decreto 619/1998, de 17 de abril de 1998 , por el que se establecen las características técnicas, el equipamiento sanitario y la dotación de personal de los vehículos de transporte sanitario por carretera. Boletín Oficial del Estado 101, del 28 de abril de 1998. (Consultado 1 diciembre de 2011). Disponible en: http://www.fomento.es/ NR/rdonlyres/834EB35C-85BF-40B1-8DF36D5848A7CC16/67666/A1412414127.pdf

21. Fundación Nacional del Transporte sanitario para LA EDUCACIÓN, COOPERACIÓN Y DESARROLLO. Libro blanco del transporte sanitario: análisis económico del sector. FNTS; 2005. (Consultado 5 de enero de 2012). Disponible en: http:// www.urjc.es/outs/Libro_Blanco/II_ANALISIS_ECONOMICO.pdf

22. LEY 16/2003, DE 28 DE MAYO, de cohesión y calidad del Sistema Nacional de Salud. Bo- letín Oficial del Estado 128, del 29 de mayo de 2003. (Consultado 1 enero de 2012). Disponible en: http://www.boe.es/boe/ dias/2003/05/29/pdfs/A20567-20588.pdf

23. VIS MA, VAN GeNT AL, editores. Road Safety Performance Indicators: Country Comparisons. Deliverable D3.7a of the EU FP6 project SafetyNet; 2007.

24. Steering Committee of the European Emergency Data Project - EMS Data-based Health Surveillance System. Grant Agreement No. SPC.2002299 under the European Community Health Monitoring Programme 1997-2002.

25. Pesqueira Alonso EE, Juliani IzQuierdo P. Unidades de soporte vital avanzado en España 2008. Mapa de situación. Emergencias 2009; 21: 269-275.

26. Grupo de Trabajo de la Sociedad Española de URGENCIAS y EMERgEncias. Protocolo de actuación y buenas prácticas en la atención sanitaria inicial al accidentado de tráfico. Ministerio de Sanidad y Política Social, 2010.

27. Generalitat de Catalunya. Pla de Govern 20112014. 3 mayo 2011. (Consultado 24 febrero de 2012). Disponible en: http://www.gencat. cat/pladegovern/cat/index.htm 